\title{
Gaps in the chromatic spectrum of face-constrained plane graphs
}

\author{
Daniel Kobler* \\ The Fields Institute \\ 222 College St. \\ Toronto, Ont. M5T 3J1 \\ Canada \\ dkobler@tmbioscience.com
}

\author{
André Kündgen ${ }^{\dagger}$ \\ Department of Computer Science \\ University of Toronto \\ Toronto, Ont. M5S 3G4 \\ Canada \\ kundgen@member.ams.org
}

Submitted: December 20, 2000; Accepted: March 23, 2001.

MR Subject Classifications: 05C15

\begin{abstract}
Let $G$ be a plane graph whose vertices are to be colored subject to constraints on some of the faces. There are 3 types of constraints: a $C$ indicates that the face must contain two vertices of a $C$ ommon color, a $D$ that it must contain two vertices of a Different color and a $B$ that Both conditions must hold simultaneously. A coloring of the vertices of $G$ satisfying the facial constraints is a strict $k$-coloring if it uses exactly $k$ colors. The chromatic spectrum of $G$ is the set of all $k$ for which $G$ has a strict $k$-coloring.

We show that a set of integers $S$ is the spectrum of some plane graph with face-constraints if and only if $S$ is an interval $\{s, s+1, \ldots, t\}$ with $1 \leq s \leq 4$, or $S=\{2,4,5, \ldots, t\}$, i.e. there is a gap at 3 .
\end{abstract}

Keywords: colorings of hypergraphs, mixed hypergraphs, planar graphs and hypergraphs, colorability, chromatic spectrum.

2000 Mathematics Subject Classification: 05C15.

All graphs and hypergraphs in this paper will be loopless, i.e. contain no edges of size 1. The problem we consider can be stated in the more general framework of mixed hypergraphs originally proposed by Voloshin $[4,5]$. A mixed hypergraph is a triple $\mathcal{H}=$ $(V, \mathcal{C}, \mathcal{D})$, where $V$ is the vertex set, and $\mathcal{C}$ and $\mathcal{D}$ are families of subsets of $V$ called the $\mathcal{C}$-edges and $\mathcal{D}$-edges. A strict $k$-coloring of a mixed hypergraph is a surjective mapping

${ }^{*}$ Supported by the Swiss National Fund and the Fields Institute.

${ }^{\dagger}$ Supported by NSERC grant of Derek Corneil and the CS Theory group. 
$c: V \rightarrow\{1,2, \ldots, k\}$ from the vertex set $V$ into a set of $k$ colors so that each $\mathcal{C}$-edge contains at least two vertices with a $\mathcal{C}$ ommon color and each $\mathcal{D}$-edge contains at least two vertices with $\mathcal{D}$ ifferent colors.

The chromatic spectrum $S(\mathcal{H})$ of a mixed hypergraph $\mathcal{H}$ is the set of all $k$ such that there is a strict $k$-coloring of $\mathcal{H}$. If $S(\mathcal{H})=\emptyset$, then $\mathcal{H}$ is uncolorable. Jiang, Mubayi, Tuza, Voloshin and West [2] showed that a set of positive integers is the chromatic spectrum of some mixed hypergraph if and only if it omits the number 1 or is an interval starting at 1 . So it is possible for the spectrum of a mixed hypergraph to have a gap, i.e. it is possible that there are integers $k<\ell<m$ such that $\mathcal{H}$ has strict $k$ and $m$-colorings, but no strict $\ell$-coloring. For example, they exhibit a mixed hypergraph $\mathcal{H}_{2,4}$ with spectrum $\{2,4\}$. This hypergraph has 6 vertices, the smallest possible for any mixed hypergraph with a gap.

Kündgen, Mendelsohn, Voloshin [3] have studied the effect that planarity can have on this coloring problem. A mixed hypergraph $(V, \mathcal{C}, \mathcal{D})$ is planar if the hypergraph with vertex-set $V$ and edge-set $\mathcal{C} \cup \mathcal{D}$ is planar. A hypergraph is said to be planar if the edges can be drawn as closed regions in the plane in such a fashion that the vertices correspond to points, where every region contains only those vertices which are in the corresponding edge, and regions only intersect in vertices. To visualize the mixed hypergraph we merely label the regions with $C, D$ or $B$, corresponding to the cases when the edge was only in $\mathcal{C}$, only in $\mathcal{D}$ or both. This is equivalent to the coloring concept given in the abstract.

Situations for which the spectrum of a planar mixed hypergraph has no gap are discussed in [3]. Since $\mathcal{H}_{2,4}$ can be seen to be non-planar, one may hope that the spectrum of every planar mixed hypergraphs is gap-free. We will show that this is almost the case. First we give an example of a planar mixed hypergraph that has a gap in its spectrum.

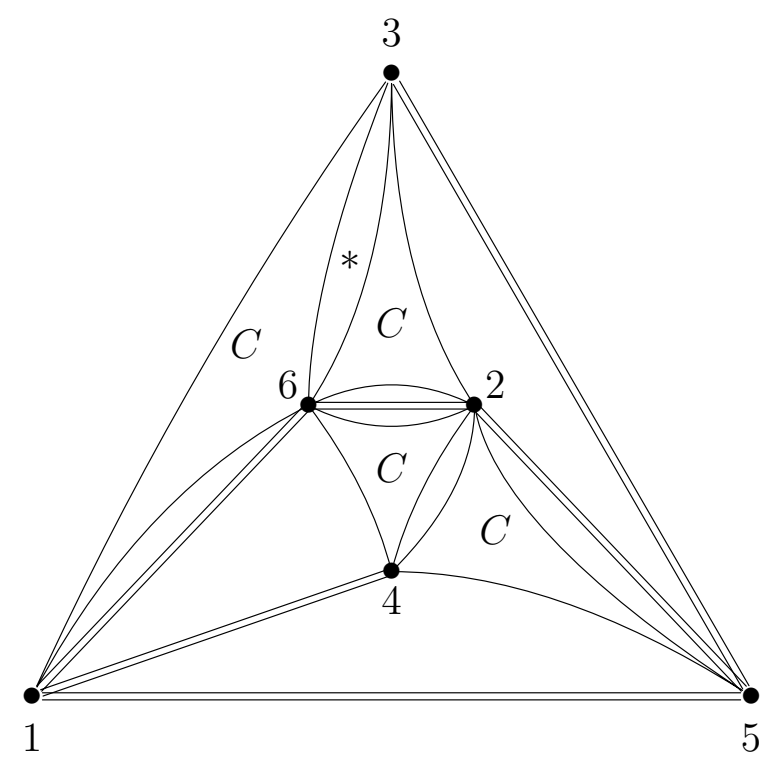

Fig. 1: $\mathcal{H}_{2,4}^{\prime}$

Lemma 1 Let $\mathcal{H}_{2,4}^{\prime}$ be the mixed hypergraph with $V=\{1, \ldots, 6\}, \mathcal{C}=\{136,236,245,246\}$, and $\mathcal{D}=\{14,15,16,25,26,35\}$. $\mathcal{H}_{2,4}^{\prime}$ is planar and has spectrum $\{2,4\}$. 
Proof. Figure 1 shows an embedding of $\mathcal{H}_{2,4}^{\prime}$ in the plane, where a $\mathcal{D}$-edge is indicated by a double-line between its two end-points. A 2-coloring is given by the partition $\{1,2,3\} \cup$ $\{4,5,6\}$ and a 4-coloring by $\{1\} \cup\{2,4\} \cup\{3,6\} \cup\{5\}$. To see that these are the only colorings consider a coloring $c$. If $c(2) \neq c(4)$, then the remaining colors are forced and we have the 2-coloring. A similar argument holds if $c(3) \neq c(6)$. If we assume that $c(2)=c(4)$ and $c(3)=c(6)$, then we get that these colors must be different, and we must have the 4-coloring.

Theorem 1 A non-empty set of positive integers $S$ is the spectrum of some planar mixed hypergraph if and only if $S$ is an interval $\{s, s+1, \ldots, t\}$ with $1 \leq s \leq 4$ or of the form $\{2,4,5, \ldots, t\}$.

Proof. Observe that a $t$-vertex planar graph $G$ is a special case of a planar mixed hypergraph with $\mathcal{C}=\emptyset$ and the $\mathcal{D}=E(G)$. The spectrum of $G$ as a mixed hypergraph is the interval $\{\chi(G), \chi(G)+1, \ldots, t\}$, where $\chi(G) \leq 4$ denotes the usual chromatic number of $G$. This shows the sufficiency of the condition when $S$ is an interval. When $S$ has a gap at 3 , consider the mixed hypergraph obtained from $\mathcal{H}_{2,4}^{\prime}$ by including the vertices $\{7, \ldots, t+2\}$ and placing them in region $*$. Also include the $\mathcal{C}$-edges $\{367,368, \ldots, 36(t+$ $2)\}$. If we have a 2-coloring on $\mathcal{H}_{2,4}^{\prime}$, then this only extends to a 2-coloring of the larger graph, whereas from the 4-coloring we obtain all other values in the spectrum.

It remains to prove the necessity of the condition. So consider a planar mixed hypergraph $\mathcal{H}$, with spectrum $S \neq \emptyset$. If $1 \in S$, then it is easy to see that $S$ forms an interval, since $\mathcal{H}$ cannot have any $\mathcal{D}$-edges. Let $c$ be a strict $t$-coloring of $\mathcal{H}$, where $t$ is the largest value in $S$. We will find a planar mixed hypergraph $\mathcal{H}^{\prime}$ with $\{4,5, \ldots, t\} \subset S\left(\mathcal{H}^{\prime}\right) \subset S$. So by the choice of $t$ it follows immediately that $S$ is of the required form.

$\mathcal{H}^{\prime}$ will have the same vertex set as $\mathcal{H}$. We will keep all edges of size 2 and since $\mathcal{H}$ is colorable these must be labeled $C$ or $D$. Now consider all regions containing at least 3 vertices. If the region is labeled $C$, then it contains vertices $u, v$ with $c(u)=c(v)$ and we replace the region by an arc from $u$ to $v$ labeled $C$, i.e. replace the $\mathcal{C}$-edge by the $\mathcal{C}$-edge $\{u, v\}$. If the edge is labeled $D$, then similarly shrink it to a $\mathcal{D}$-edge $\{u, v\}$ consisting of vertices with $c(u) \neq c(v)$. If the region is labeled with $B$, then it contains vertices $u, v, w$ such that $c(u)=c(v) \neq c(w)$ and we replace the corresponding $\mathcal{C}$-edge by $\{u, v\}$ and the $\mathcal{D}$-edge by $\{v, w\}$. The mixed hypergraph $\mathcal{H}^{\prime}$ we obtain is planar and still has $c$ as a strict $t$-coloring. Furthermore, in obtaining $\mathcal{H}^{\prime}$ from $\mathcal{H}$, no coloring constraints were lost (some of them were even sharpened), so that every coloring of $\mathcal{H}^{\prime}$ is a coloring of $\mathcal{H}$.

Observe that we now have a planar hypergraph with all edges of size 2 , some of which are labeled $C$ and others $D$. If we contract all edges labeled $C$, then we obtain a loopless planar graph $G$ and there is a 1-1 correspondence between strict colorings of $G$ and strict colorings of $\mathcal{H}^{\prime}$. Since the spectrum of $G$ must be the interval $\{\chi(G), \chi(G)+1, \ldots, t\}$, we obtain that $\{4,5, \ldots, t\} \subset S\left(\mathcal{H}^{\prime}\right)$ as desired.

In [3] it was shown that if a planar mixed hypergraph is uncolorable, then it must contain edges of size 2 . In other words, the "bad behavior" of uncolorability depends on 
edges of size 2. Similarly, we believe that if a planar mixed hypergraph has a gap in the spectrum, then it must also contain edges of size 2 .

By a reduction similar to that in Theorem 1 it suffices to consider the case when all edges are of size 3, i.e. the mixed hypergraph is 3-uniform. It was shown in [3] that every 3 -uniform planar mixed hypergraph is 2-colorable. Therefore it would be enough to show that every 3-uniform 4-colorable planar mixed hypergraph is also 3-colorable. Notice that from the proof technique used in [3], it would even follow that every 3-uniform planar mixed hypergraph on $\geq 5$ vertices is 3 -colorable if the following question could be answered in the affirmative:

Is $K_{4}$ the only cubic planar graph without cut-edges all of whose 2-factors are Hamiltonian?

This question has been investigated with only partial success and an infinite family of counterexamples can be found if one drops the planarity requirement [1]. There may also be a more straightforward approach to show that the spectrum of 3-uniform planar mixed hypergraphs is gap-free.

\section{Acknowledgments}

The authors would like to thank Radhika Ramamurthi for suggestions on the manuscript.

\section{References}

[1] M. Funk, B. Jackson, D. Labbate and J. Sheehan, 2-Factor Hamiltonian Graphs, hardcopy preprint 2000115, University of Aberdeen (2000).

[2] T. Jiang, D. Mubayi, Zs. Tuza, V. Voloshin and D.B. West, Chromatic spectrum is broken, 6th Twente Workshop on Graphs and Combinatorial Optimization, 26-28 May, 1999, H.J. Broersma, U. Faigle and J.L. Hurink (eds.). University of Twente, May, 1999, 231-234.

[3] A. Kündgen, E. Mendelsohn, V. Voloshin, Colouring planar mixed hypergraphs, Electron. J. Combin. 7 (2000), \#R60.

[4] V. Voloshin, The mixed hypergraphs, Computer Science Journal of Moldova 1 (1993), $45-52$.

[5] V. Voloshin, On the upper chromatic number of a hypergraph, Australasian Journal of Combinatorics 11 (1995), 25-45. 